\title{
Three-component elastic wave band-gap material
}

\author{
Zhengyou Liu, ${ }^{*}$ C. T. Chan, and Ping Sheng \\ Physics Department, Hong Kong University of Science and Technology, Clear Water Bay, Hong Kong, China
}

(Received 24 October 2001; revised manuscript received 31 January 2002; published 11 April 2002)

\begin{abstract}
Using multiple-scattering theory, we consider the elastic wave band-gap properties of three-component composites consisting of a periodic arrangement of coated spheres inside an embedding medium. We found that the elastic wave band-gap properties can be tuned continuously from a resonance gap to a Bragg gap just by varying the elastic properties of one component. If the coating material is soft compared to the core and the matrix, the elastic wave band gap is essentially a resonance gap. If the coating material is stiff, the elastic wave gap is derived from Bragg scattering. These systems are ideal for realizing elastic wave gap and wave localization as well as understanding the underlying mechanism.
\end{abstract}

DOI: 10.1103/PhysRevB.65.165116

PACS number(s): 62.30.+d

\section{INTRODUCTION}

In the past decade, there have been intense activities on photonic band-gap ${ }^{1}$ systems because of their novel physical properties and many potential applications in the photonics age. Elastic wave can also have forbidden gaps, but it has received less attention partly because it is a more complex mathematical problem. Another reason is that elastic wave band-gap material is usually conceived to have a length scale at least a few times that of the wavelength, implying gigantic structures for lower frequencies and making the application rather difficult. Previous considerations are mainly focused on two-component systems ${ }^{2}$ and absolute elastic wave band gaps are rather difficult to realize for two-component solid materials in three dimensions.

There are two mechanisms that can lead to a forbidden gap in classical waves. One is Bragg scattering in periodic systems and the opening of a Bragg gap at the Brillouin zone (BZ) boundary. ${ }^{1}$ The existence of an absolute gap requires the overlap of the Bragg gaps in all directions. Symmetry, periodicity, and orderness of the periodic system are all important. The frequency of the spectral gap is of the order of $c / a$, where $c$ is wave speed and $a$ is the lattice constant. The photonic band gap, as it was originally perceived, is a Bragg gap that overlaps in all $4 \pi$ radians. The other mechanism is derived from localized resonances. Examples are polariton gaps when light couples with optical phonons in ionic crystals. The frequency of a resonance gap is dictated by the frequency of the resonance, and is independent of orderness, periodicity and, symmetry unless there is a high concentration of resonating units so that they couple strongly with each other. Electromagnetic resonances derived from elementary excitations such as polariton or plasmon are usually associated with strong absorption, and are thus not very useful for creating photonic gaps. The situation is different for elastic waves in at least two respects. First, the dissipation can be very small in certain mechanical excitations, making resonance a possible mechanism for the creation a elastic wave band gap. Second, we shall show in this paper that it is possible to tune the spectral gap "continuously" between a Bragg gap and a resonance gap in threecomponent elastic systems, while such manipulations would be very difficult for electromagnetic waves.
In order to realize a complete elastic wave band gap and tune its property from a Bragg gap to a resonance gap (as well as somewhere in between) we employ a threecomponent configuration. All that is required is to vary the elastic property of one of the component relative to the other two. Our calculation for these three-component systems not only elucidates the gap formation mechanisms, but also provides a recipe that guarantees the existence of elastic wave band gaps and a good chance to realize localization. The configuration consists of a spherical core, a concentric coating of another material, and an embedding matrix of a third material. The core does not need to be spherical, but it makes the calculations more tractable. All the calculations are performed with a multiple-scattering method, which gives excellent agreement with experimental results. ${ }^{3}$ The details of the multiple-scattering theory can be found elsewhere, ${ }^{3}$ and will be outlined in the following section so that various symbols and notations are clearly defined. We note that the method allows us to solve accurately the elastic wave equations when the Lame constants and the densities of the constituent components are specified. We fix the core material as $\mathrm{Au}$ and the matrix as $\mathrm{Si}$, and only vary the coating layer material. In the results presented, the radius of the Au core and the total radius of coated sphere $(\mathrm{Au}+$ coating) are fixed at a ratio of 13 to 18 . We note that this particular ratio has no special significance except that we have to choose some numbers to do our numerical calculations. Other ratios can have the same qualitative effect. Unless otherwise indicated, all frequencies are normalized to $d / 2 \pi c_{t}$, where $d$ is the diameter of the coated sphere and $c_{t}$ is the transverse sound speed. We will see that when the coating is soft compared with the elastic constants of the core and the matrix material, the gap is a resonance gap. If the coating is stiff, the gap becomes a Bragg gap.

\section{MULTIPLE-SCATTERING METHOD FOR ELASTIC WAVE BAND STRUCTURE}

We shall consider a composite medium that contains a host matrix and embedded scatterers. The incident wave for scatterer $i$ may be expressed as 


$$
\mathbf{u}^{i n}\left(\mathbf{r}_{i}\right)=\sum_{l m \sigma} a_{l m \sigma}^{i} \mathbf{J}_{l m \sigma}\left(\mathbf{r}_{i}\right)
$$

and the scattered wave by scatterer $i$ may be expressed as

$$
\mathbf{u}^{s c}\left(\mathbf{r}_{i}\right)=\sum_{l m \sigma} b_{l m \sigma}^{i} \mathbf{H}_{l m \sigma}\left(\mathbf{r}_{i}\right),
$$

where, $\mathbf{r}_{i}$ is measured from the center of scatterer $i$, and $\mathbf{J}_{l m \sigma}(\mathbf{r}), \mathbf{H}_{l m \sigma}(\mathbf{r})$ are defined as

$$
\begin{gathered}
\mathbf{J}_{l m 1}(\mathbf{r})=\frac{1}{\alpha} \nabla\left[j_{l}(\alpha r) Y_{l m}(\hat{\mathbf{r}})\right], \\
\mathbf{J}_{l m 2}(\mathbf{r})=\frac{1}{\sqrt{l(l+1)}} \nabla \times\left[\mathbf{r} j_{l}(\beta r) Y_{l m}(\hat{\mathbf{r}})\right], \\
\mathbf{J}_{l m 3}(\mathbf{r})=\frac{1}{\sqrt{l(l+1)} \beta} \nabla \times \nabla \times\left[\mathbf{r} j_{l}(\beta r) Y_{l m}(\hat{\mathbf{r}})\right],
\end{gathered}
$$

and

$$
\begin{gathered}
\mathbf{H}_{l m 1}(\mathbf{r})=\frac{1}{\alpha} \nabla\left[h_{l}(\alpha r) Y_{l m}(\hat{\mathbf{r}})\right], \\
\mathbf{H}_{l m 2}(\mathbf{r})=\frac{1}{\sqrt{l(l+1)}} \nabla \times\left[\mathbf{r} h_{l}(\beta r) Y_{l m}(\hat{\mathbf{r}})\right], \\
\mathbf{H}_{l m 3}(\mathbf{r})=\frac{1}{\sqrt{l(l+1)} \beta} \nabla \times \nabla \times\left[\mathbf{r} h_{l}(\beta r) Y_{l m}(\hat{\mathbf{r}})\right],
\end{gathered}
$$

$$
\mathbf{H}_{l^{\prime \prime} m^{\prime \prime} \sigma^{\prime \prime}}\left(\mathbf{r}_{j}\right)=\sum_{l m \sigma} G_{l^{\prime \prime} m^{\prime \prime} \sigma^{\prime \prime} l m \sigma}^{i j} \mathbf{J}_{l m \sigma}\left(\mathbf{r}_{i}\right)
$$

where $G_{l^{\prime \prime} m^{\prime \prime} \sigma^{\prime \prime} l m \sigma}^{i j}=G_{l^{\prime \prime} m^{\prime \prime} \sigma^{\prime \prime} l m \sigma}\left(\mathbf{R}_{i}-\mathbf{R}_{j}\right)$, the latter is given

by

where $\mathbf{r}_{i}$ and $\mathbf{r}_{j}$ refer to the position of the same spatial point measured from scatterers $i$ and $j$, respectively. With $\mathbf{R}_{i(j)}$ denoting the position of scatterer $i(j)$, we have $\mathbf{r}_{j}=\mathbf{r}_{i}+\mathbf{R}_{i}$ $-\mathbf{R}_{j}$. It can be shown that

$$
G_{l m \sigma l^{\prime} m^{\prime} \sigma^{\prime}}(\mathbf{R})= \begin{cases}X_{l m l^{\prime} m^{\prime}}^{\alpha}(\mathbf{R}), & \sigma=\sigma^{\prime}=1, \\ \sum_{\mu} c(l 1 l m-\mu \mu) X_{l m-\mu l^{\prime} m^{\prime}-\mu^{\prime}}^{\beta}(\mathbf{R}) c\left(l^{\prime} 1 l^{\prime} m^{\prime}-\mu \mu\right), & \sigma=\sigma^{\prime}=2,3, \\ -i\left(\frac{2 l^{\prime}+1}{l^{\prime}+1}\right)^{1 / 2} \sum_{\mu} c(l 1 l m-\mu \mu) X_{l m-\mu l^{\prime}-1 m^{\prime}-\mu^{\prime}}^{\beta}(\mathbf{R}) c\left(l^{\prime}-11 l^{\prime} m^{\prime}-\mu \mu\right), & \sigma \neq \sigma^{\prime} ; \sigma, \sigma^{\prime} \neq 1,\end{cases}
$$

where, "c" are Clebsch-Gordan coefficients, and $X_{l m l^{\prime} m^{\prime}}^{\kappa}(\mathbf{R})$ is defined as

$$
\begin{aligned}
X_{l m l^{\prime} m^{\prime}}^{\kappa}(\mathbf{R})= & 4 \pi \sum_{l^{\prime \prime}} i^{l^{\prime}+l^{\prime \prime}-l} C_{l^{\prime} m^{\prime} l^{\prime \prime} m-m^{\prime}}^{l m} h_{l^{\prime \prime}} \\
& \times(\kappa R) Y_{l^{\prime \prime} m-m^{\prime}}(\hat{\mathbf{R}}),
\end{aligned}
$$

and $C_{l^{\prime} m^{\prime} l^{\prime \prime} m^{\prime \prime}}^{l m}$ is defined as

$$
C_{l^{\prime} m^{\prime} l^{\prime \prime} m^{\prime \prime}}^{l m}=\iint_{S} Y_{l m}(\Omega) Y_{l^{\prime} m^{\prime}}^{*}(\Omega) Y_{l^{\prime \prime} m^{\prime \prime}}^{*}(\Omega) d \Omega .
$$

where $\alpha=\omega \sqrt{\rho /(\lambda+2 \mu)}, \beta=\omega \sqrt{\rho / \mu}$ (where $\rho, \lambda$, and $\mu$ mectively, the mass density and two Lame constants of $h_{l}(x)$ is the spherical Hankel function of the first kind. Acon a nally incident wave, $\mathbf{u}^{\text {in(0)}}\left(\mathbf{r}_{i}\right)$, which may be expanded as

The second part is the sum of all the scattered waves except that from scatterer $i$, given by

$$
\mathbf{u}^{i n}\left(\mathbf{r}_{i}\right)-\mathbf{u}^{i n(0)}\left(\mathbf{r}_{i}\right)=\sum_{j \neq i} \sum_{l^{\prime \prime} m^{\prime \prime} \sigma^{\prime \prime}} b_{l^{\prime \prime} m^{\prime \prime} \sigma^{\prime \prime}}^{j} \mathbf{H}_{l^{\prime \prime} m^{\prime \prime} \sigma^{\prime \prime}}\left(\mathbf{r}_{j}\right)
$$




$$
\begin{aligned}
\sum_{j l^{\prime} m^{\prime} \sigma^{\prime}} & {\left[\delta_{i j} \delta_{l l^{\prime}} \delta_{m m^{\prime}} \delta_{\sigma \sigma^{\prime}}\right.} \\
& \left.-\sum_{l^{\prime \prime} m^{\prime \prime} \sigma^{\prime \prime}} t_{l^{\prime \prime} m^{\prime \prime} \sigma^{\prime \prime} l^{\prime} m^{\prime} \sigma^{\prime}} G_{l^{\prime \prime} m^{\prime \prime} \sigma^{\prime \prime} l m \sigma}^{i j}\right] a_{l^{\prime} m^{\prime} \sigma^{\prime}}^{j}=a_{l m \sigma}^{i(0)}
\end{aligned}
$$

This is the final equation for a multiple-scattering system. For a finite and/or disordered system, we must solve this equation in order to investigate the system response to external perturbations. The normal modes of the system may be obtained by solving the following secular equation, which in the absence of external incident wave becomes:

$$
\operatorname{det}\left|\delta_{i j} \delta_{l l^{\prime}} \delta_{m m^{\prime}} \delta_{\sigma \sigma^{\prime}}-\sum_{l^{\prime \prime} m^{\prime \prime} \sigma^{\prime \prime}} t_{l^{\prime \prime} m^{\prime \prime} \sigma^{\prime \prime} l^{\prime} m^{\prime} \sigma^{\prime}}^{j} G_{l^{\prime \prime} m^{\prime \prime} \sigma^{\prime \prime} l m \sigma}^{i j}\right|=0 \text {. }
$$

For a periodic system, Eq. (13) may be transformed to

$$
\begin{aligned}
& \operatorname{det}\left|\delta_{s s^{\prime}} \delta_{l l^{\prime}} \delta_{m m^{\prime}} \delta_{\sigma \sigma^{\prime}}-\sum_{l^{\prime \prime} m^{\prime \prime} \sigma^{\prime \prime}} t_{l^{\prime \prime} m^{\prime \prime} \sigma^{\prime \prime} l^{\prime} m^{\prime} \sigma^{\prime}}^{s^{\prime}} G_{l^{\prime \prime} m^{\prime \prime} \sigma^{\prime \prime} l m \sigma}^{s s^{\prime}}(\mathbf{k})\right| \\
& \quad=0
\end{aligned}
$$

where $s$ and $s^{\prime}$ label the scatterers in the unit cell with position vectors $\mathbf{o}_{s}$ and $\mathbf{o}_{s^{\prime}}$, and $G_{l^{\prime \prime} m^{\prime \prime} \sigma^{\prime \prime} l m \sigma}^{s s^{\prime}}(\mathbf{k})$ is defined as

$$
G_{l^{\prime \prime} m^{\prime \prime} \sigma^{\prime \prime} l m \sigma}^{s s^{\prime}}(\mathbf{k})=\sum_{\mathbf{R}} G_{l^{\prime \prime} m^{\prime \prime} \sigma^{\prime \prime} l m \sigma}\left(\mathbf{o}_{s}-\mathbf{o}_{s^{\prime}}-\mathbf{R}\right) \exp (i \mathbf{k} \cdot \mathbf{R}) .
$$

where the sum $\Sigma_{\mathbf{R}}$ is over all lattice sites. The solution of Eq. (15) gives the band structure of an elastic periodic system.

We now outline the procedure to obtain the Mie scattering matrix of a spherical scatterer with coating. Let the inner radius of the coating (i.e., the bare core sphere radius) be $a$, and the outer radius be $b$. The material constants for the host medium are the mass density $\rho_{1}$ and the Lame constants $\lambda_{1}$ and $\mu_{1}$. The corresponding material constants for the coating material are denoted as $\rho_{2}, \lambda_{2}$, and $\mu_{2}$ and those for the sphere are denoted as $\rho_{3}, \lambda_{3}$, and $\mu_{3}$. The displacement field in the host medium may be expressed as

$$
\mathbf{u}_{1}(\mathbf{r})=\sum_{l m \sigma}\left[a_{l m \sigma} \mathbf{J}_{l m \sigma}^{(1)}(\mathbf{r})+b_{l m \sigma} \mathbf{H}_{l m \sigma}^{(1)}(\mathbf{r})\right]
$$

where the superscripts (1) on the right-hand side, which are all omitted for convenience in the previous subsection, index the host medium. Similarly, the displacement in the coating layer may be expressed as

$$
\mathbf{u}_{2}(\mathbf{r})=\sum_{l m \sigma}\left[c_{l m \sigma} \mathbf{J}_{l m \sigma}^{(2)}(\mathbf{r})+d_{l m \sigma} \mathbf{H}_{l m \sigma}^{(2)}(\mathbf{r})\right]
$$

while that in the core sphere may be expressed as

$$
\mathbf{u}_{3}(\mathbf{r})=\sum_{l m \sigma} e_{l m \sigma} \mathbf{J}_{l m \sigma}^{(3)}(\mathbf{r}) .
$$

The strain tensor in the $i$ th medium $(i=1,2,3)$ is expressed as

$$
\boldsymbol{\epsilon}_{i}=\frac{1}{2}\left(\nabla \mathbf{u}_{i}+\mathbf{u}_{i} \nabla\right),
$$

and the stress and the strain tensors are related by

$$
\boldsymbol{\tau}_{i}=\lambda_{i}\left(\boldsymbol{\epsilon}_{i}: \mathbf{I}\right) \mathbf{I}+2 \mu_{i} \boldsymbol{\epsilon}_{i} .
$$

Displacement and normal stress continuity at the interface requires that

$$
\begin{gathered}
\left.\mathbf{u}_{3}(\mathbf{r})\right|_{r=a}=\left.\mathbf{u}_{2}(\mathbf{r})\right|_{r=a}, \\
\left.\boldsymbol{\tau}_{3}(\mathbf{r}) \cdot \mathbf{r}\right|_{r=a}=\left.\boldsymbol{\tau}_{2}(\mathbf{r}) \cdot \mathbf{r}\right|_{r=a}, \\
\left.\mathbf{u}_{2}(\mathbf{r})\right|_{r=b}=\left.\mathbf{u}_{1}(\mathbf{r})\right|_{r=b}, \\
\left.\boldsymbol{\tau}_{2}(\mathbf{r}) \cdot \mathbf{r}\right|_{r=b}=\left.\boldsymbol{\tau}_{1}(\mathbf{r}) \cdot \mathbf{r}\right|_{r=b}
\end{gathered}
$$

The boundary conditions lead to 12 linear equations involving coefficients $\left\{a_{l m \sigma}\right\},\left\{b_{l m \sigma}\right\},\left\{c_{l m \sigma}\right\},\left\{d_{l m \sigma}\right\}$, and $\left\{e_{l m \sigma}\right\}$, from which we obtain the relation between coefficients $A$ $=\left\{a_{l m \sigma}\right\}$ and $B=\left\{b_{l m \sigma}\right\}$ formally as

$$
B=T A,
$$

where $T=\left\{t_{l m \sigma l^{\prime} m^{\prime} \sigma^{\prime}}\right\}$ is the scattering matrix as defined. It can be shown that, for spherical scatterer (with or without coating), the scattering matrix is independent of $m$, and diagonal in $l$ :

$$
t_{l m \sigma l^{\prime} m^{\prime} \sigma^{\prime}}=t_{\sigma \sigma^{\prime}} \delta_{l l^{\prime}},
$$

moreover, only five elements of $t_{\sigma \sigma^{\prime}}$ are nonzero, the $3 \times 3$ matrix $\left\{t_{\sigma \sigma^{\prime}}\right\}$ looks like

$$
\left[\begin{array}{lll}
L L & 0 & L N \\
0 & M M & 0 \\
L N & 0 & N N
\end{array}\right]
$$

where symbol $L$ denotes the longitudinal modes $(\sigma=1), M$ denotes the first transverse modes $(\sigma=2)$, and $N$ denotes the second transverse modes $(\sigma=3)$. The meanings of the five nonzero elements are straightforward, for example, $L L$ stands for the conversion from $L$ mode to $L$ mode during the scattering procedure. It shows that the $L$ modes and the $N$ modes are coupled to each other, while the $M$ modes are decoupled.

\section{RESULTS}

We first consider the case in which the coating layer is chosen to be lead, a material that is softer than both Au (the core) and $\mathrm{Si}$ (the embedding matrix). The material parameters used in the calculation are $\rho=19.5 \mathrm{~g} / \mathrm{cm}^{3}, c_{l}$ $=3.36 \mathrm{~km} / \mathrm{s}, \quad c_{l} / c_{t}=2.71$ for $\mathrm{Au} ; \quad \rho=2.33 \mathrm{~g} / \mathrm{cm}^{3}, \quad c_{l}$ $=8.95 \mathrm{~km} / \mathrm{s}, c_{l} / c_{t}=1.67$ for $\mathrm{Si}$; and $\rho=11.4 \mathrm{~g} / \mathrm{cm}^{3}, c_{l}$ $=2.16 \mathrm{~km} / \mathrm{s}, c_{l} / c_{t}=2.51$ for $\mathrm{Pb}$; where $\rho, c_{l}$, and $c_{t}$ are, respectively, the density, the longitudinal and transverse sound velocity. We show the elastic wave band structure with 


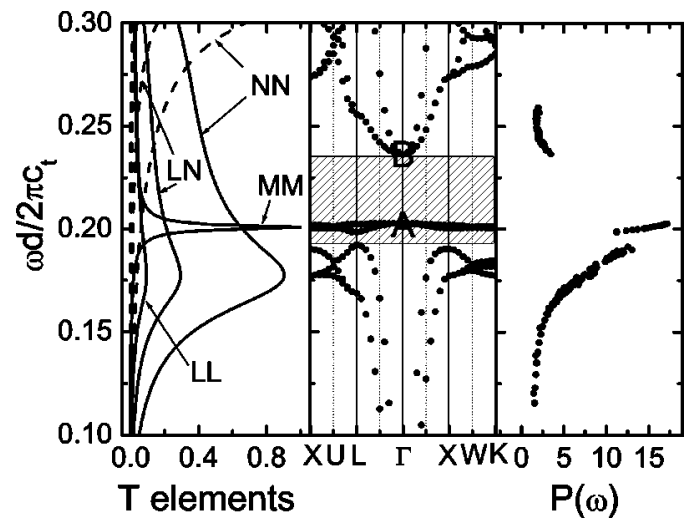

FIG. 1. Middle panel: The elastic wave band structures of leadcoated spheres arranged in the fcc structure (filling fraction is $10 \%$ ). The gap is marked by the shaded area. Right panel: Inverse participation ratio for normal modes (see text for definition). Left panel: $T$-matrix elements for $l=1$ (solid lines) and $l=2$ (dotted lines). The symbols $L, M, N$ represent different polarizations. See text for definition.

lead as the coating layer in the middle panel of Fig. 1. The spheres are arranged in an fcc structure and occupies $10 \%$ of the volume. A sizable absolute gap is seen with frequency at about 0.2 , in units of $d / 2 \pi c_{t}$. The left panel shows the absolute values of the $T$-matrix elements as a function of frequency, defined by Eq. (22) in the previous section. Since the $T$ matrix is independent of $m$ and is diagonal in $l$, we can consider the $T$-matrix elements for each $l$ separately, and the polarization index $\sigma$ is the only index that varies. For the frequencies of interest, we found that only $l=1$ and 2 have significant amplitudes. We see from the middle panel that there is a flat band near 0.2. Comparison with the left panel shows that the flat band is derived from $l=1 M M$ resonances ( $M$ polarized incident wave scattered into a $M$ polarized outgoing wave). We further note that the strong $L L, N L$, and $N N$ resonances (with $l=1$ ) that peak at about 0.175 units give rise to the set of relatively flat bands in the band structure. With these comparisons, the mechanism of the gap formation emerges. The coated spheres have strong resonances, as show by the peaks of the $T$-matrix elements on the left panel. Away from these resonances, the three-component system behaves like an effective medium for longwavelength elastic waves. For $\omega<0.15$, the dispersions are essentially straight lines, with the slopes corresponding to the speeds of the elastic wave for each polarization. When the frequency reaches those of the $l=1$ resonances, the coupling between the linear dispersion and the flat resonances of the individual spheres opens a gap, which accounts for the gap that is observed above 0.2 . The $M M$ resonances, poorly coupled to other modes, give rise to a set of nearly dispersionless bands that split off from the top of the lower edge of gap. It is clear that the gap is derived from localized resonances of the coated spheres.

By examining the amplitude of the displacement fields at the zone center of the modes marked $A$ and $B$, we found that the displacement field of mode $B$ is mainly localized in the Au core. It is basically the vibration mode of the Au core as a whole with some noticeable elastic deformation. We also



FIG. 2. The gap and the midgap frequencies of lead-coated spheres in the fcc structure plotted as a function of the filling ratio, compared with other structures. The midgap frequencies of a variety of other structures $(\mathrm{sc}=$ simple cubic, dia $=$ diamond $)$ are marked by crosses. See text for details.

observe nonzero displacement fields in the Si matrix, indicating the coupling of the host elastic wave with the Au core. The displacement fields of modes labeled $A$ are essentially a libration type deformation of the $\mathrm{Pb}$ coating, with nearly no coupling to the $\mathrm{Si}$ matrix. Consider the ratio $P$ defined as

$P(\omega)=[D(\omega)]^{-1} \sum_{n, k} \delta\left(\omega-\omega_{n, k}\right) V \int u_{n, k}^{4} d \vec{r}\left(\int u_{n, k}^{2} d \vec{r}\right)^{-2}$,

where $u$ is the amplitude of the displacement fields of the eigenmodes, $n, k$ are band and $k$-point indices, and the integration is performed over the unit cell volume $(V)$, and $D(\omega)$ is the density of modes. In the right panel of Fig. 1, we plot the ratio $P$ as a function of frequency. The value of $P(\omega)$ should vary from a small number for extended waves to a large value if the normal mode amplitude is strongly concentrated within some positions within the unit cell. It is clear from this plot that the A modes are very localized, which accounts for the flatness of the band at 0.2 . The very flat dispersion inside a spectral gap, together with the strong concentration of displacement amplitude in real space, makes the modes in band $A$ an excellent candidate for observing classical wave Anderson localization if a small amount of disorder is introduced. The fairly flat modes just below the absolute gap should also be easily localized by disorder. The classical wave localization can be observed at very low frequencies $(\lambda \gg d)$ if the cladding is very soft. It is particularly interesting since one would normally expect homogenization to be valid in that regime (if one ignores the existence of resonances).

If the gap is derived from localized resonances, we expect that the property of the gap should be independent of the arrangement of the spheres. We have calculated the gap size and midgap frequencies with the spheres arranged in the fcc structure as a function of the filling ratio of the coated spheres. The bars in Fig. 2 mark the size of the gap and the line in the middle marks the midgap frequencies for the fcc structure. In the same figure, we plot the calculated gap size and midgap frequencies for a variety of structures, including hexagonal close packed (hcp), simple cubic (sc), diamond 


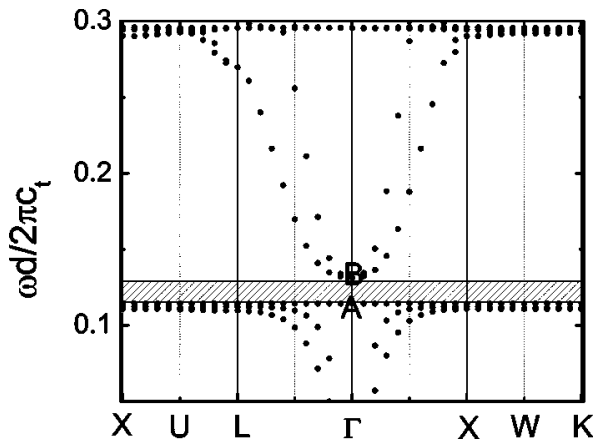

FIG. 3. The elastic wave band structure of plastic coated spheres in the fcc structure with a filling fraction of $10 \%$. The absolute gap is marked by the shaded area.

(dia), body centered cubic (bcc), with the midgap frequencies of these structures marked by crosses. We do see a rather universal behavior, consistent with the notion that localized resonance of the individual sphere is the main mechanism for gap formation. We note that the gap starts to disappear at higher filling fractions. This is not surprising since the description based on the resonance of an individual sphere is less accurate when the packing ratio is high and the individual resonances start to couple with each other. For the Bragg scattering mechanism, the gap typically peaks at some intermediate filling fractions. The diminishing of the gap at high filling fraction is a signature that Bragg mechanism is also playing a role in determining the gap properties at higher filling fractions, where the resonances of the individual spheres are coupled.

Can we tune the system so that the localized resonance behavior is even more dominant in governing the gap properties? To this end, we make the coating even softer and use plastic as the prototype of very soft material. The material parameters for plastic are taken to be $\rho=1.19 \mathrm{~g} / \mathrm{cm}^{3}, c_{l}$ $=2.75 \mathrm{~km} / \mathrm{s}, c_{l} / c_{t}=2.29$.

In Fig. 3, we plot the elastic wave band structure for such a configuration with the spheres arranged in a periodic fcc structure. The filling ratio of the coated spheres is $10 \%$. The qualitative features are rather similar to the band structure with $\mathrm{Pb}$ as the coating layer (Fig. 1), except that the dispersion near the gap is even more typical of that of dispersionless localized resonance, and the softer coating moves the resonance gap to a lower frequency (slightly above 0.1 units). This is confirmed by analyzing the displacement patterns of the modes near the $\Gamma$ point. We found that the displacement field for modes marked as $B$ in Fig. 3 is localized in the core, corresponding to the vibration of the Au core as a whole with very small elastic deformation. The displacement fields of modes marked as $A$ are completely localized in the soft plastic cladding layer.

In Fig. 4, we plot the size of the gap and the midgap frequencies for a variety of structures as a function of the filling ratio, with the midgap frequencies marked by solid squares and the size of the gap marked by the bar. The gap and midgap frequencies for the fcc structure for a range of filling ratio from $5 \%$ to $60 \%$ are plotted in the same figure for comparison, with the upper (lower) line marking the upper (lower) edge of the fcc gaps, while the middle line marks

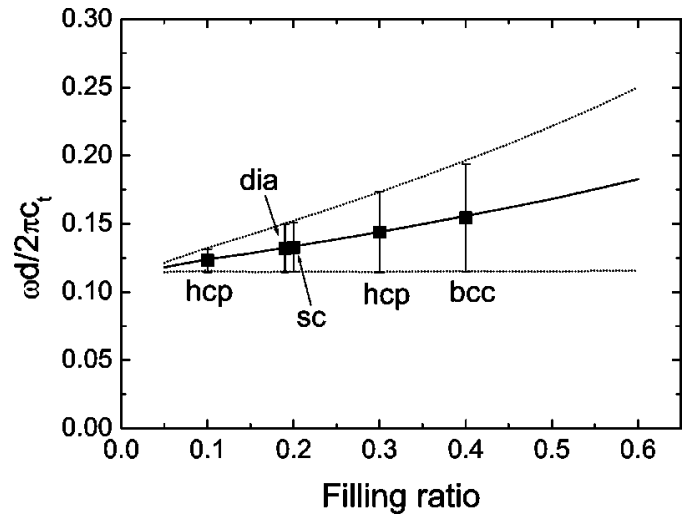

FIG. 4. The gap and midgap frequencies of the fcc structure for a range of filling ratios with plastic as the coating layer. The upper (lower) line is the upper (lower) edge of the fcc gap, while the middle line marks the fcc midgap frequency. The midgap frequencies for a variety of structures (dia $=$ diamond, $\mathrm{sc}=$ simple cubic) are marked by squares and the size of the gaps by the bars. Note the universal behavior.

the fcc midgap frequencies. We note that property of the gap (its midgap frequency and the size of the gap) is independent of the geometrical arrangement and is only governed by the filling ratio. In addition, the size of the gap increases monotonically as the filling ratio increases. These two are strong evidences that the gap is the result of localized resonances and because of this, the results become "universal" when we normalize the frequency to the diameter of the sphere. For localized resonances, the scattering cross section is determined by the property of the scatterer and hence the diameter of the sphere is the most important length scale. For Bragg scattering, it would have been the lattice constant that is the relevant length scale. Also, for Bragg scattering, the symmetry and the shape of the BZ is important, and the gap property is strongly dependent on the geometric arrangement. The fact that the size of the gap increases monotonically with the filling ratio is another characteristic of localized resonance. If Bragg scattering is the dominant mechanism, the size of the gap always peaks at some intermediate filling ratio.

We note further that the lower edge of the gap is pinned by the highly localized libration mode of the plastic coating. It has virtually no dependence on filling ratio, consistent with the high degree of localization of this libration mode. Since the gap is derived from localized resonances, its existence is guaranteed and is independent of the details of the structure and should persist even for a random arrangement of spheres. ${ }^{4}$ The "hard-soft-hard" three-component configuration thus offers us a recipe for absolute elastic wave band gap, with the added advantage that the gap frequency can be very low if we can find a very soft cladding.

To demonstrate that we can tune the system within the same three-component configuration so that Bragg scattering becomes dominant, we change the coating to a material that is stiffer. We will use steel as the prototypical stiff coating, with material parameters $\rho=7.67 \mathrm{~g} / \mathrm{cm}^{3}, c_{l}=6.01 \mathrm{~km} / \mathrm{s}$, $c_{l} / c_{t}=1.86$.

The fcc elastic wave band structures for $10 \%$ filling frac- 


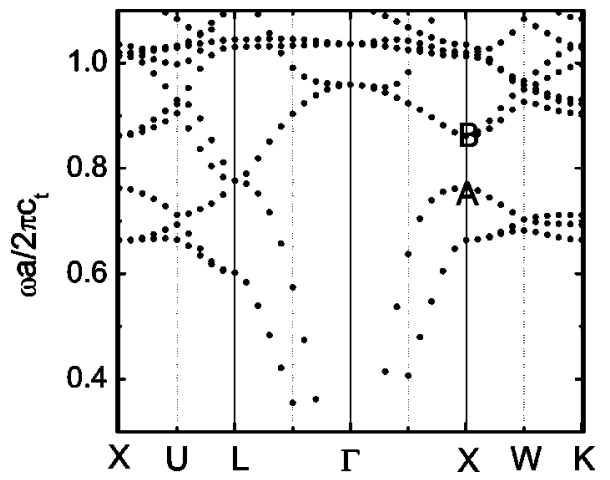

FIG. 5. The band structure for steel coated spheres arranged in the fcc structure (10\% filling fraction).

tion is plotted in Fig. 5. The frequency here is normalized to $a / 2 \pi c_{t}$, where $a$ is the fcc lattice constant. We note immediately that there is no absolute gap, but we observe a directional gap at the $X$ point. Since steel is harder than the Au core, the resonance due to the vibration of the Au core surrounded by a soft coating no longer exists and the dispersion is now governed by Bragg scattering, which opens spectral gaps at the Brillouin-zone boundaries. Since the directional gaps are derived from Bragg scattering, we choose to normalize the frequency in Fig. 5 to the lattice constant $a$ rather than the diameters of the spheres. An absolute gap is an exception rather than the rule for the Bragg scattering mechanism unless the directional gaps in all solid angles overlap. For our system, we observe gaps along the $X-W-K$ directions at about 0.8 units, but there are propagating modes along the $\Gamma-L-U$ directions in the same frequency range. It is conceivable that an absolute gap can be found by adjusting the thickness of the coating and filling ratio, but the point here is to show that the spectral gap formation mechanism is dominated by Bragg scattering if the coating is stiff. When we examine the field patterns for modes labeled $A$ and $B$, they are very different from the cases with $\mathrm{Pb}$ or plastic coating. Both modes are typical extended Bloch waves with mode $A$ being more localized in the core, while mode $B$ is a very extended mode with mode amplitudes peaking outside the core. We have also calculated $P(\omega)$ [see Eq. 25] as a function of frequency and the values are small, again confirming that the modes are extended.

\section{SUMMARY}

In conclusion, a three-component system with a coated gold core embedded in a Si matrix exhibits elastic wave gap such that the gap formation mechanism can be tuned continuously from a resonance gap to a Bragg gap by changing the softness of the coating. These systems thus serve as an excellent prototype for understanding the mechanisms leading to classical wave gaps and their interplay. If the coating layer is sufficiently soft, localized vibrational resonances always guarantees an absolute elastic wave band gap, with a good chance to observe classical wave localization if disorder is introduced. We emphasize that it is the relative softness of the coating layer relative to the core and the matrix that is important. We could, for example, replace the core by copper and see qualitatively similar results. We also note that some of us have proposed to use coated spheres (glass spheres coated with a layer of metal and an outer layer of dielectrics) to create photonic band gaps..$^{5}$ The mechanism for creating the photonic gap using metal coated spheres is fundamentally different from the concept introduced here. Here, the elastic wave gap is created by resonances where the core plays an active role, while for the case of photons, ${ }^{5}$ the glass core merely serves a passive support for the metallic coating. The metallic coating squeezes all the field energy into the voids between the spheres, creating a situation in which a robust photonic band gap can be formed.

\section{ACKNOWLEDGMENTS}

This work was supported by RGC-Hong Kong through HKUST6145/99P and HKUST6143/00P.
*Permanent address: Physics Dept., Wuhan University, Wuhan 430072, China.

${ }^{1}$ See, e.g., E. Yablonovitch, Phys. Rev. Lett. 58, 2059 (1987); S. John, ibid. 58, 2486 (1987); J. Joannopoulos, R.D. Meade, and J. Winn, Photonic Crystals (Princeton University Press, Princeton, NJ, 1995).

${ }^{2}$ See, e.g., M.S. Kushwaha, P. Halevi, P.L. Dobrzynski, and B. Djafari-Rouhani, Phys. Rev. Lett. 71, 2022 (1993); E.N. Economou and M. Sigalas, J. Acoust. Soc. Am. 95, 1734 (1994); M. Kafesaki, M.M. Sigalas, E.N. Economou, Solid State Commun. 96, 285 (1995); R. Martinez-Sala, J. Sancho, J.V. Sanchez, V. Gomez, J. Llinares, and F. Meseguer, Nature (London) 378, 241 (1995); M.S. Kushwaha, P. Halevi, G. Martynez, L. Dobrzynski, and B. Djafari-Rouhani, Phys. Rev. B 49, 2313 (1994); M. Si- galas and E.N. Economou, Europhys. Lett. 36, 241 (1996); J.V. Sanchez-Perez, D. Caballero, R. Martinez-sala, C. Rubio, J. Sanchez-Dehesa, F. Meseguer, J. Llinares, and F. Galvez, Phys. Rev. Lett. 80, 5325 (1998); F.R. Montero de Espinosa, E. Jimenez, and M. Torres, ibid. 80, 1208 (1998); A.D. Klironomos and E.N. Economou, Solid State Commun. 105, 327 (1998); I.E. Psarobas, N. Stefanou, and A. Modinos, Phys. Rev. B 62, 278 (2000).

${ }^{3}$ Z.Y. Liu, C.T. Chan, P. Sheng, A.L. Goertzen, and J.H. Page, Phys. Rev. B 62, 2446 (2000).

${ }^{4}$ Z.Y. Liu, X.X. Zhang, Y. Mao, Y.Y. Zhu, Z. Yang, C.T. Chan, and P. Sheng, Science 289, 1734 (2000).

${ }^{5}$ W.Y. Zhang, X.Y. Lei, Z.L. Wang, D.G. Zheng, W.Y. Tam, C.T. Chan, and P. Sheng, Phys. Rev. Lett. 84, 2853 (2000). 